\title{
Efectos contextuales asociados a la variabilidad del riesgo de depresión en adultos mayores, Antioquia, Colombia, 2012
}

\author{
Doris Cardona1, Alejandra Segura², Ángela Segura1', María Osley Garzón ${ }^{1}$ \\ 1 Facultad de Medicina, Universidad CES, Medellín, Colombia \\ 2 Hospital Pablo Tobón Uribe, Medellín, Colombia
}

\begin{abstract}
Introducción. La depresión es un trastorno del afecto en el que se pierde el interés de vivir y se alteran de manera negativa los ámbitos laboral, afectivo, relacional y personal. Sin embargo, poco se sabe sobre si hay asociación entre el lugar de residencia y dicha alteración del estado de ánimo.

Objetivo. Determinar los efectos del municipio y la manzana de residencia en la variabilidad del riesgo de depresión en los adultos mayores del departamento de Antioquia, Colombia, en el 2012.

Materiales y métodos. Se llevó a cabo un estudio descriptivo multinivel de fuente secundaria con 4.060 registros de adultos mayores del departamento de Antioquia. Se valoraron características demográficas, sociales y funcionales en el cálculo de las razones de momios (odds ratio, OR) crudas y ajustadas, para buscar asociación entre el riesgo de depresión medida con la escala de depresión geriátrica de Yesavage y las variables de contexto (municipio y manzana de residencia) en la construcción del modelo, utilizando los métodos de cadenas de Markov Monte Carlo (Markov Chain Monte Carlo, MCMC); la bondad de ajuste de los modelos se evaluó mediante el criterio de información de la desviación (Deviance Information Criterion, DIC).

Resultados. La prevalencia del riesgo de depresión fue de 29,5 \% y se encontró asociación con la edad mayor de 75 años, el sexo femenino, la residencia en zona rural y la condición de viudez. De igual forma, se encontró riesgo de experimentar ansiedad, capacidad funcional moderada y alto riesgo de desnutrición. Se encontró asociación entre el municipio y la manzana de residencia, y el riesgo de depresión.

Conclusiones. El municipio y la manzana de residencia aportaron $10 \%$ de la variabilidad total del riesgo de depresión en los adultos mayores. Esta información es importante para fomentar la participación y adhesión de los adultos mayores a grupos comunitarios.
\end{abstract}

Palabras clave: depresión, distribución espacial de la población, anciano, análisis multinivel, ansiedad, geografía.

doi: http://dx.doi.org/10.7705/biomedica.v35i1.2381

\section{Contextual effects associated with depression risk variability in the elderly, Antioquia, Colombia, 2012}

Introduction: Depression is an affective disorder where interest in living is lost and functional areas like work, feelings, personal life, and relationships are negatively altered. However, little has been said about the association between place of residence and this mood disorder.

Objective: To determine the effects of the municipality and block of residence on the depression risk variability in the elderly in the Department of Antioquia, Colombia, in 2012.

Materials and methods: This was a multilevel descriptive study of secondary source records of 4,060 elderly people from the Department of Antioquia. Demographic, social and functional characteristics were assessed to calculate raw and adjusted odds ratios and find an association between the risk of depression, as measured by Yesavage's Geriatric Depression Scale, and context variables (municipality and block of residence) in the design of the model. We used Markov chain Monte Carlo estimation methods and the deviance information criterion to assess goodness of fit.

Results: The prevalence of the risk of depression was $29.5 \%$ and we found an association with ages over 75 years, being female, residing in rural areas, and widowhood. Additionally, they had a higher risk of anxiety, moderate functional capacity and malnutrition. The municipality and block of residence were associated with this risk in the elderly.

Conclusions: The municipality and block of residence had a $10 \%$ contribution toward the total variability in the risk of depression for the elderly. This information is important to encourage participation and adherence of the elderly to community groups.

Key words: Depression, residence characteristics, aged, multilevel analysis, anxiety, geography. doi: http://dx.doi.org/10.7705/biomedica.v35i1.2381

\footnotetext{
Contribución de las autoras:

Todas las autoras participaron en el proyecto y la elaboración del artículo.
} 
Entre los fenómenos demográficos que tendrán lugar durante el siglo XXI, el envejecimiento de la población es uno de los más relevantes por los cambios que genera en las estructuras sociales, económicas y culturales de los países. Según la Organización Mundial de la Salud (OMS), la proporción de personas mayores de 60 años está aumentando más rápidamente que cualquier otro grupo de edad en casi todos los países. El envejecimiento de la población puede considerarse un éxito de las políticas de salud pública y del desarrollo socioeconómico, pero también constituye un reto para la sociedad, que debe garantizar el mejoramiento de la salud física y mental y en el estado funcional de las personas mayores, así como propiciar su participación social y su seguridad (1).

El crecimiento de la población adulta mayor trae consigo un aumento de las enfermedades mentales relacionadas con la edad, como las demencias y la depresión, esta última considerada como el principal problema de salud mental que el mundo enfrentará en el futuro (2). La depresión es el trastorno psiquiátrico más frecuente entre los mayores; su presencia reduce la calidad de vida, incrementa las enfermedades físicas y acorta la vida media (3).

Según Erikson (4), lo ideal en la etapa final de la vida sería experimentar la "integridad del yo frente a la desesperación", pues es la etapa en la que el individuo puede sentir que ha merecido la pena vivir, estar satisfecho con las decisiones tomadas y contemplar la vida como un todo significativo. Por el contrario, una resolución negativa de la crisis de esta etapa puede desembocar en el arrepentimiento y el remordimiento por decisiones equivocadas de la persona en el pasado, que le generan desilusión y pesadumbre y el deseo de dar marcha atrás y desandar lo andado (5).

En esta edad, el sentimiento de desesperanza y el trastorno depresivo aparecen con mayor frecuencia en los hombres debido al cambio de roles, a su menor participación en la toma de decisiones, a la falta de autoridad y la inactividad forzada, entre otros factores. Las mujeres mayores, por su parte, están más protegidas, pues cuentan con un círculo

\section{Correspondencia:}

Doris Cardona Arango, Facultad de Medicina, Universidad CES, Calle $10 \mathrm{~A} \mathrm{~N}^{\circ} 22-04$, Medellín, Colombia

Teléfono (574) 444 0555; fax: (574) 2666046

doris.cardona@gmail.com, dcardona@ces.edu.co

Recibido: 19/05/14; aceptado: 27/10/14 de intereses más amplio y una red de relaciones informales más estables, que le ofrecen mayor gratificación psicológica (6).

La depresión es la enfermedad más común y la principal causa de suicidio entre las personas de edad avanzada. Si bien el $13 \%$ de la población de los Estados Unidos tiene más de 65 años, es en este grupo en el que se da el $20 \%$ de los suicidios. La más alta tasa de suicidio en el mundo en cualquier grupo de edad se encuentra entre los hombres que tienen 75 años o más, y el $90 \%$ de quienes se suicidan tienen, por lo menos, una dolencia mental diagnosticable o un trastorno relacionado con el abuso de drogas (7). La apatía o las quejas vagas por malestares físicos, también pueden enmascarar síntomas de depresión e incidir considerablemente en el riesgo de ataques cardíacos, así como de fracturas de cadera e infecciones graves (7).

Las encuestas han demostrado su utilidad en la detección oportuna de la población con riesgo de presentar depresión, así como para evaluar los posibles riesgos asociados (8). La escala más adecuada para adultos mayores es la escala de depresión geriátrica de Yesavage, ya que brinda una sensibilidad de $85 \%$ y una especificidad de $95 \%(9)$.

Si bien existen escalas de medición del riesgo de padecer este trastorno, son pocos los estudios que involucran los aspectos contextuales, por lo que en este se buscó determinar los efectos del municipio de residencia en la variabilidad de los niveles del riesgo de depresión de los adultos mayores del departamento de Antioquia, Colombia, en el año 2012, con el fin de verificar la hipótesis de que el riesgo de depresión en los adultos mayores del departamento es diferente según el municipio de residencia.

\section{Materiales y métodos}

Se realizó un estudio observacional y descriptivo transversal entre 4.060 adultos mayores de 60 años en una población total de 576.080 asentada en 417 manzanas (en promedio, 9,74 personas por manzana).

Las encuestas se hicieron en 37 municipios $(11,27$ manzanas por municipio, en promedio) de las nueve subregiones del departamento de Antioquia y en la ciudad capital: Bajo Cauca, Magdalena Medio, Medellín, Nordeste, Norte, Occidente, Oriente, Suroeste, Urabá y Valle de Aburrá. En cada subregión se tomó una muestra representativa de 
adultos mayores, se seleccionaron cuatro municipios por muestreo aleatorio simple, además de Medellín, donde se tomaron cuatro comunas de la ciudad mediante el mismo tipo de muestreo, a las cuales se les asignó el mismo peso de los municipios frente al total.

El tamaño de la muestra en cada subregión se calculó con la fórmula de Fleiss para poblaciones finitas en el programa Epidat 3.1, con un nivel de confianza del $95 \%$, un error de muestreo del 1,6\%, una proporción de buen estado de salud del $50 \%$ y un efecto de diseño de 1,0.

La muestra de adultos mayores dentro de la subregión se seleccionó mediante muestreo probabilístico, sistemático y aleatorio, por conglomerados y en etapas sucesivas. En cada subregión, los adultos mayores se seleccionaron en los municipios, considerados como las unidades terciarias de muestreo, en tanto que las unidades secundarias fueron las manzanas, seleccionadas con muestreo aleatorio simple, y las unidades primarias fueron los hogares donde residía cada adulto mayor.

La encuesta se aplicó exclusivamente a los adultos mayores, y en ella se indagó sobre las características demográficas, establecidas como variables independientes, y sobre el riesgo de depresión, que fue la variable dependiente (10), tomada en forma cuantitativa para el análisis multinivel, y en forma cualitativa (con riesgo y sin él) para la asociación con las demás variables estudiadas.

Para determinar el riesgo de depresión, se utilizó la escala de valoración geriátrica de depresión de Yesavage (11), la cual se emplea en personas mayores pero no es un sustituto de la entrevista diagnóstica a cargo de profesionales de salud mental. De todas maneras, es una herramienta útil y rápida, ya probada en Colombia (9), que se usa en el ámbito clínico y en estudios de población (12).

Las variables independientes incluyeron el sexo, la edad, el área de procedencia y el estado civil. También, se emplearon las escalas para detectar el riesgo de ansiedad (Escala de Ansiedad y Depresión de Goldberg, EADG) (13), la capacidad funcional (índice de Katz) (14) y el riesgo nutricional (escala Determine) (15), según las recomendaciones del Centro Latinoamericano y Caribeño de Demografía (CELADE, División de Población) (16).

Con los datos se hizo un análisis univariado utilizando el programa SPSS ${ }^{\circledR}$, versión 21 (licencia de la Universidad CES) para el cálculo de las medidas estadísticas y de frecuencia. A continuación, se calcularon las razones de prevalencia (RP) y se determinaron las variables asociadas al riesgo de padecer depresión mediante la prueba estadística de ji al cuadrado con un intervalo de confianza del $95 \%\left(\mathrm{IC}_{95 \%}\right)$; la asociación estadísticamente significativa se fijó con valores de p menores de $5 \%$.

Con el fin de probar si existían diferencias en el riesgo de esta condición individual que pudieran explicarse con base en las variables contextuales de la manzana y el municipio de residencia, se ajustó un modelo logístico de tres niveles: en el primer nivel se consideraron los 4.060 adultos mayores, cantidad que permitía calcular la prevalencia del riesgo de depresión sin efectos aleatorios; en el segundo nivel se ubicaron las manzanas, con sus variables de contexto dentro de cada municipio seleccionado, y en el tercer nivel, establecido como máximo nivel de exposición al riesgo, se incluyeron los 37 municipios para calcular los coeficientes de asociación, el ajuste de las relaciones encontradas, la varianza $\left(\mathrm{S}^{2}\right)$ y el coeficiente de partición de varianza (CPV).

Se ajustaron tres modelos: un modelo nulo con el cual se estimó el riesgo promedio de depresión en toda la población y los respectivos componentes de varianza para cada uno de los tres niveles; un modelo con las variables individuales, y un tercer modelo que incluyó las variables de manzana y municipio. Este análisis se hizo con el programa MLwiN, versión 2.28 (licencia de la Universidad CES), utilizando métodos MCMC, los cuales proporcionan estimaciones más sólidas (17). La bondad de ajuste de los modelos se evaluó aplicando el criterio de información de la desviación (DIC), según el cual el modelo con el menor DIC se considera el mejor; igualmente, la asociación estadísticamente significativa se estableció en valores de $p$ menores de $5 \%$.

Se deja constancia de que este proyecto se ajustó a las consideraciones éticas de la Resolución 8430 de 1993 del Ministerio de Salud y se clasificó como una investigación de riesgo mínimo; para hacer las pruebas psicológicas a los adultos mayores se obtuvo su consentimiento informado. Los impedimentos cognitivos se establecieron como criterio de exclusión. El proyecto fue avalado por el Comité Institucional de Ética de la Universidad CES mediante el Acta 53 del 1 de octubre de 2012.

\section{Resultados}

\section{Características demográficas}

Los adultos mayores encuestados presentaron las siguientes características demográficas: $62 \%$ eran mujeres y $38 \%$, hombres, y 69,1 \% tenían 
menos de 75 años. Predominaron las personas que residían en el área urbana de sus municipios y la mayoría eran casados, aunque en el caso de las mujeres predominó el estado de viudez (cuadro 1).

Según la escala utilizada, el puntaje promedio de riesgo de depresión fue de 4,41 ( $\pm 3,19)$ : el más frecuente fue de 3; el mínimo de 0 y el máximo de 18. Una vez categorizada, la prevalencia de riesgo de depresión fue de 29,5\%: el 20,5\% presentó un riesgo leve y el $9,0 \%$, un riesgo mayor. En cuanto a los resultados por sexo, las mujeres presentaron un mayor riesgo que los hombres (cuadro 1).

Un poco más de la mitad de los encuestados no registró riesgo de ansiedad, pero las mujeres lo presentaron más que los hombres. Ocho de cada diez adultos mayores se consideraban independientes para realizar las actividades de la vida diaria y dos de cada tres tenían alto riesgo de desnutrición, sin mayores diferencias por sexo (cuadro 1).

\section{Factores asociados al riesgo de depresión}

De los adultos encuestados, el 29,5\% tenía algún riesgo de padecer depresión. El $30,5 \%$ de las mujeres lo presentaron frente al $27,8 \%$ de los hombres. Por cada mujer con riesgo de presentar depresión, hubo 0,9 hombres con el mismo riesgo; es decir, la prevalencia en los hombres fue menor $(\mathrm{RP}=0,91 \quad[0,820 ; 1,004])$; sin embargo, no se encontró asociación estadísticamente significativa entre estas variables ni con el área de procedencia, pero el riesgo en los residentes en el área rural fue mayor, por lo que el hecho de vivir en un área urbana podría considerarse como un factor de protección ( $\mathrm{RP}=0,903[0,764 ; 1,068]$ ) (cuadro 2).

En cuanto a la edad de los encuestados, clasificados en menores y mayores de 75 años, se encontró que $36,6 \%$ de los mayores de 75 años presentaba riesgo de depresión y se evidenció una asociación estadísticamente significativa entre la edad y dicho riesgo. También, se encontró asociación estadística entre la condición civil de los adultos mayores viudos y el riesgo de depresión, siendo mayor en los viudos $(36,3 \%)$ y menor en los casados $(23,2 \%)$ (cuadro 2).

El riesgo de depresión se encontró asociado con el riesgo de ansiedad (48,3\%), con la dependencia funcional moderada $(77,8 \%)$ y con el alto riesgo de desnutrición $(35,4 \%)$ (cuadro 2).

Cuadro 1. Distribución porcentual de los adultos mayores según características demográficas y por sexo, Antioquia, 2012

\begin{tabular}{|c|c|c|c|c|c|c|}
\hline \multirow[t]{3}{*}{ Característica demográfica } & \multicolumn{4}{|c|}{ Sexo } & \multicolumn{2}{|c|}{ Total } \\
\hline & \multicolumn{2}{|c|}{ Hombre } & \multicolumn{2}{|c|}{ Mujer } & \multirow[b]{2}{*}{$\mathbf{n}$} & \multirow[b]{2}{*}{$\%$} \\
\hline & $\mathbf{n}$ & $\%$ & $\mathbf{n}$ & $\%$ & & \\
\hline \multicolumn{7}{|l|}{ Área de procedencia } \\
\hline Urbana & 1.386 & 90,9 & 2.284 & 92,8 & 3.670 & 92,1 \\
\hline Rural & 138 & 9,1 & 176 & 7,2 & 314 & 7,9 \\
\hline Estado civil & & 15,6 & 414 & 16,5 & 654 & 16,2 \\
\hline Soltero & 240 & & & & & \\
\hline Casado & 787 & 51,2 & 801 & 31,9 & 1.588 & 39,2 \\
\hline Viudo & 223 & 14,4 & 974 & 38,8 & 1.197 & 29,6 \\
\hline Separado/divorciado & 123 & 8,0 & 212 & 8,4 & 335 & 8,3 \\
\hline Unión libre & 164 & 10,7 & 111 & 4,4 & 275 & 6,8 \\
\hline \multicolumn{7}{|l|}{ Riesgo de depresión } \\
\hline Normal & 1.113 & 72,2 & 1.751 & 69,5 & 2.864 & 70,5 \\
\hline Riesgo leve & 316 & 20,5 & 517 & 20,5 & 833 & 20,5 \\
\hline Riesgo establecido & 112 & 7,3 & 251 & 10,0 & 363 & 8,9 \\
\hline \multicolumn{7}{|l|}{ Riesgo de ansiedad } \\
\hline Sin riesgo & 967 & 63,2 & 1.293 & 51,8 & 2.260 & 56,1 \\
\hline Con riesgo & 562 & 36,8 & 1.204 & 48,2 & 1.766 & 43,9 \\
\hline \multicolumn{7}{|l|}{ Capacidad funcional } \\
\hline Dependencia grave & 20 & 1,3 & 41 & 1,6 & 61 & 1,5 \\
\hline Dependencia moderada & 13 & 0,8 & 32 & 1,3 & 45 & 1,1 \\
\hline Dependencia leve & 196 & 12,8 & 329 & 13,2 & 525 & 13,0 \\
\hline Independencia & 1.303 & 85,1 & 2.096 & 83,9 & 3.399 & 84,3 \\
\hline \multicolumn{7}{|l|}{ Riesgo de desnutrición } \\
\hline Buen estado nutricional & 181 & 12,3 & 272 & 11,3 & 453 & 11,7 \\
\hline Riesgo moderado & 289 & 19,6 & 492 & 20,4 & 781 & 20,1 \\
\hline Riesgo alto & 1.003 & 68,1 & 1.647 & 68,3 & 2.650 & 68,2 \\
\hline
\end{tabular}


Cuadro 2. Asociación entre las características demográficas y el riesgo de depresión en los adultos mayores, Antioquia, 2012

\begin{tabular}{|c|c|c|c|c|c|c|c|c|}
\hline \multirow[t]{3}{*}{ Variables demográficas } & \multicolumn{4}{|c|}{ Riesgo de depresión } & \multirow[t]{3}{*}{$\chi^{2}$} & \multirow[t]{3}{*}{$p$} & \multirow[t]{3}{*}{$\mathbf{R P}$} & \multirow[t]{3}{*}{$I C_{95 \%}$} \\
\hline & \multicolumn{2}{|c|}{ Sin riesgo } & \multicolumn{2}{|c|}{ Con riesgo } & & & & \\
\hline & $\mathbf{n}$ & $\%$ & $\mathbf{n}$ & $\%$ & & & & \\
\hline \multicolumn{9}{|l|}{ Sexo } \\
\hline Hombre & 1.113 & 72,2 & 428 & 27,8 & 3,389 & 0,066 & 1,000 & - \\
\hline Mujer & 1.751 & 69,5 & 768 & 30,5 & & & 1,039 & 0,998-1,082 \\
\hline \multicolumn{9}{|l|}{ Edad } \\
\hline Menos de 75 años & 2.094 & 74,7 & 711 & 25,3 & 73,784 & $0,000^{*}$ & 1,000 & - \\
\hline 75 años o más & 770 & 61,4 & 485 & 38,6 & & & 1,217 & $1,159-1,278$ \\
\hline \multicolumn{9}{|l|}{ Área de procedencia } \\
\hline Urbana & 2.599 & 70,8 & 1.071 & 29,2 & 2,158 & 0,142 & 1,000 & - \\
\hline Rural & 210 & 66,9 & 104 & 33,1 & & & 1,059 & $0,977-1,148$ \\
\hline \multicolumn{9}{|l|}{ Estado civil } \\
\hline Soltero & 445 & 68,0 & 209 & 32,0 & 60,113 & $0,000^{*}$ & 1,000 & - \\
\hline Casado & 1.220 & 76,8 & 368 & 23,2 & & & 0,642 & $0,525-0,786$ \\
\hline Viudo & 762 & 63,7 & 435 & 36,3 & & & 1,215 & $0,993-1,488$ \\
\hline Separado/divorciado & 231 & 69,0 & 104 & 31,0 & & & 0,959 & $0,722-1,273$ \\
\hline Unión libre & 198 & 72,0 & 77 & 28,0 & & & 0,828 & $0,607-1,129$ \\
\hline \multicolumn{9}{|l|}{ Riesgo de ansiedad } \\
\hline Sin riesgo & 1.938 & 85,8 & 322 & 14,2 & 556,238 & $0,000^{*}$ & 1,000 & - \\
\hline Con riesgo & 913 & 51,7 & 853 & 48,3 & & & 1,659 & $1,581-1,740$ \\
\hline \multicolumn{9}{|l|}{ Capacidad funcional } \\
\hline Depresión grave & 23 & 37,7 & 38 & 62,3 & 155,458 & $0,000^{*}$ & 4,708 & $2,789-7,946$ \\
\hline Depresión moderada & 10 & 22,2 & 35 & 77,8 & & & 9,973 & $4,916-20,223$ \\
\hline Dependencia leve & 294 & 56,0 & 231 & 44,0 & & & 2,239 & $1,854-2,703$ \\
\hline Independencia & 2.516 & 74,0 & 883 & 26,0 & & & 1,000 & - \\
\hline \multicolumn{9}{|l|}{ Riesgo de desnutrición } \\
\hline Buen estado nutricional & 407 & 89,8 & 46 & 10,2 & 195,712 & $0,000^{*}$ & 1,000 & - \\
\hline Riesgo moderado & 656 & 84,0 & 125 & 16,0 & & & 1,686 & $1,176-2,416$ \\
\hline Riesgo alto & 1.713 & 64,6 & 937 & 35,4 & & & 4,840 & $3,532-6,632$ \\
\hline
\end{tabular}

* $p<0,005 ;$ RP: razón de prevalencia

\section{Efectos contextuales en el riesgo de depresión}

Con el fin de estudiar los efectos contextuales en el riesgo de padecer depresión, las variables individuales se conjugaron con las variables de manzana y municipio de residencia en un análisis multinivel. Se calculó un modelo nulo, el cual mostró un puntaje promedio de 4,429 de riesgo de depresión, con una variabilidad de 0,154 , valor que representa el error estándar (EE) del parámetro, como se ve a continuación.

\section{Ecuación 1:}

Depresión Codpersona, Codman, Codmuni $\sim \mathrm{N}(X B, \Omega)$

Depresión ${ }_{\text {Codpersona, Codman, Codmuni }}=4.429(0.154)$ Const

$-2 * \operatorname{loglikelihood}($ IGLS Deviance $)=20587.735$ (4060 of 4060 cases in use)

Posteriormente, se incorporaron las variables de los demás niveles, es decir, persona, manzana y municipio de residencia.

\section{Ecuación 2:}

Depresión Codpersona, Codman, Codmuni $\sim \mathrm{N}(X B, \Omega)$
Depresión Codpersona, Codman, Codmuni $=\beta_{\text {0Codpersona, Codman, Codmuni }}$ Const $\beta_{0 \text { Codpersona, Codman, Codmuni }}=4.429(0.154)+v_{0 \text { Codmuni }}+u_{0 \text { Codman, Codmuni }}$ $+e_{0 \text { Codpersona, Codman, Codmuni }}$

$-2 * \operatorname{loglikelihood}($ IGLS Deviance $)=20587.735$ (4060 of 4060 cases in use), donde, v=municipio; $u=$ manzana; e=persona.

Finalmente, el modelo nulo se estableció de la siguiente manera.

\section{Ecuación 3:}

Depresión $_{i j k} \sim \mathrm{N}(X B, \Omega)$

Depresión $_{i j k}=\beta_{0 i j k}$ Const

$\beta_{0 i j k}=4.429(0.154)+v_{0 k}+u_{0 j k}+e_{0 i j k}$

$\left[v_{0 k}\right] \sim \mathrm{N}\left(0, \Omega_{v}\right): \Omega_{v}=[0.801(0.217)]$

$\left[u_{0 j k}\right] \sim \mathrm{N}\left(0, \Omega_{u}\right): \Omega_{u}=[0.496(0.114)]$

$\left[e_{0 i j k}\right] \sim \mathrm{N}\left(0, \Omega_{e}\right): \Omega_{e}=[8.786(0.205)]$

$-2 * \log$ likelihood(IGLS Deviance $)=20587.735$ (4060 of 4060 cases in use),

Al analizar la varianza en los tres niveles (persona, manzana y municipio), se encontró que el riesgo de depresión variaba principalmente entre las personas 
$(8,786$, error estándar $=0,205)$; sin embargo, se observó una varianza significativa en el riesgo de depresión entre los municipios $(0,801, \mathrm{EE}=0,217)$, $y$ entre las manzanas $(0,496, E E=0,114)$. Esto demuestra la pertinencia de llevar a cabo un análisis multinivel, ya que hay porcentajes de varianza en el riesgo de depresión en los adultos mayores que pueden explicarse por las características del municipio y la manzana de residencia.

Los resultados muestran que, aunque el puntaje promedio de riesgo en todos los municipios fue de 4,429 puntos $\left(S^{2}=0,154\right)$, se observaron diferencias importantes entre ellos. Por ejemplo, en el 2,5\% de los municipios el menor puntaje promedio de depresión fue de 2,68 puntos o menos, mientras que en el 2,5\% de los municipios con mayor riesgo, el puntaje fue de 6,16 puntos o más.

En el 10,083 \% de la población de estudio, la varianza total del riesgo de depresión respondió a variables de contexto relacionadas con el municipio y la manzana de residencia del adulto mayor. Al descomponer el coeficiente de participación de varianza (CPV), se encontró que el 7,9\% de la variabilidad del riesgo de depresión de los adultos mayores se explicaba por variables de contexto relacionadas con el municipio de residencia: el $5,0 \%$ de esta variación respondía a las condiciones de la manzana dentro del municipio y el $87,1 \%$ se atribuyó a las variables de persona (cuadro 3).

\section{Discusión}

La Organización Mundial de la Salud reportó una cifra ponderada de depresión en la población adulta mayor del 10,4\%, con variaciones según el lugar de residencia como, por ejemplo, las siguientes: Santiago de Chile, donde la prevalencia de depresión correspondió al 29,5\%, similar a la encontrada

Cuadro 3. Varianza y porcentaje de varianza explicado del riesgo de depresión en los adultos mayores según variables de persona, manzana y municipio de residencia, Antioquia, 2012

\begin{tabular}{lcrc}
\hline Modelos & \multicolumn{2}{c}{$\begin{array}{c}\text { Prevalencia de riesgo de depresión } \\
\text { (puntaje >5) }\end{array}$} \\
\cline { 2 - 4 } & Varianza (EE) & p & $\begin{array}{r}\text { \% de varianza } \\
\text { no explicada }\end{array}$ \\
\hline Modelo 1 (nulo) & & & \\
Persona (e) & $8,786(0,205)$ & $<0,001$ & 87,137 \\
Manzana (u) & $0,496(0,114)$ & 0,002 & 4,919 \\
Municipio (v) & $0,801(0,217)$ & $<0,001$ & 7,944 \\
Varianza total & 10,083 & & \\
\hline
\end{tabular}

EE: error estándar en este estudio; Rio de Janeiro, con una prevalencia del 15,8\%; Shanghai, donde fue de alrededor de $4 \%$, e Ibadan, en Nigeria, con 4,2\% (18).

En México, la encuesta nacional de epidemiología psiquiátrica señalaba que $9,1 \%$ de los adultos mayores había padecido un trastorno afectivo alguna vez en la vida; de este porcentaje, el 4,8 \% correspondió a episodios de depresión mayor o menor (19). Estos resultados difieren de lo reportado por Tapia, et al., en México en el 2000 (20), quienes encontraron una prevalencia de depresión leve de $32,5 \%$, de depresión media de $44,5 \%$ y de depresión grave de 2,5\%.

En esta investigación se observó una frecuencia de riesgo de depresión más alta en el sexo femenino, aunque esta asociación no fue significativa. El predominio de la prevalencia de depresión en mujeres está ampliamente documentado; en ellas es de, aproximadamente, el doble de la observada en los hombres; la mayor parte de los antecedentes, nacionales e internacionales, coinciden en esta diferencia $(8,9,17,21)$; sin embargo, algunas de las divergencias de los estudios con relación al sexo, podrían explicarse por las variaciones en las distribuciones socioculturales de las poblaciones. Las investigaciones señalan que la depresión es más frecuente en la mujer que en el hombre, independientemente de la edad, dado que la razón oscila entre 2 y 3 dependiendo del tipo de factores determinantes sociales, del país y del momento en que se hace la medición (22).

En cuanto a la edad, al igual que en la presente investigación, diversos estudios coinciden en afirmar que la prevalencia de depresión aumenta con la edad (23). Pattern, Sedwack y Russel aseguran que, a medida que aumenta la edad, disminuye la posibilidad de ser diagnosticado debido a que se presta mayor atención a las enfermedades médicas que a las mentales en este grupo de edad y a que el cuidado de los trastornos afectivos en mayores de 60 años se considera un reto para el sistema de salud (24). En el 2001, Gómez, et al., señalaron que en Colombia el riesgo de depresión en los adultos mayores aumentaba con la edad (25).

El riesgo de depresión es mayor en los adultos mayores que se encuentran solos. En este estudio, el estado civil mostró diferencias significativas con relación al riesgo de depresión, pero difirieron de lo reportado en el 2000 por Tapia, et al., (20), quienes encontraron un mayor riesgo en los casados, seguidos de los viudos y los solteros. Castro, et al., (8) aseguraron que en la literatura científica 
hay reportes que indican que las personas que no tienen una relación conyugal, los que tienen predisposición genética y los que han tenido una pérdida sentimental reciente, son blanco perfecto para la depresión.

Con respecto a la autonomía del adulto mayor, al igual que lo hallado por algunos autores $(26,27)$, en este estudio se encontró que una mayor prevalencia de riesgo de depresión se asoció con la dependencia física para la realización de las actividades diarias, situación que parece clara teniendo en cuenta que dicha limitación, así como la condición de dependencia, la pérdida de control, la sensación de vulnerabilidad y el sentimiento de ser una carga para el otro, generan malestar y frustración en el adulto mayor (28). Estos resultados coinciden con los reportados por Estrada, et al., en el 2008 (21), quienes encontraron que las actividades funcionales se asociaron con la presencia de depresión, siendo los adultos con déficit funcional los que tuvieron mayor prevalencia de síntomas depresivos.

En el estudio de Gómez-Restrepo, et al., realizado en Colombia, se encontró que la dependencia en la ejecución de las actividades diarias y la discapacidad fueron factores asociados con la depresión en $62 \%$ (funcionalidad medida por la escala de Katz) (25), la cual también mostró una estrecha relación con las enfermedades crónicas. Además, cerca de $80 \%$ de los sujetos de 70 años o más sufrían una enfermedad crónica y 50 \% experimentaba algún trastorno que obstaculizaba el funcionamiento independiente, por lo que la depresión en la vejez se consideró una consecuencia psíquica de los procesos físicos de debilitamiento de las funciones vitales (29).

Hay una considerable superposición entre los perfiles de riesgo de ansiedad y depresión en los adultos mayores, lo que sugiere un enfoque dimensional en la interrelación entre la ansiedad y la depresión para mejorar el reconocimiento y los programas preventivos de salud mental, generar una comprensión más clara y diferenciar los factores etiológicos de cada una (30).

Generalmente, los factores biológicos parecen ser más importantes en la predicción de la depresión, razón por la cual existen pocos estudios que relacionen los efectos del vecindario, el barrio y los municipios de residencia con el riesgo de depresión en los adultos mayores, aunque sí los ha habido que evidencian la relación entre la pérdida de la capacidad funcional (31) y la variación con respecto a trastornos mentales en la población general, con una varianza de 14,4\% (32).
Entre las limitaciones de este estudio vale mencionar su carácter descriptivo transversal, lo cual no permitió establecer una relación de causalidad, así como el uso de una fuente que sugería el riesgo de depresión y no de enfermedad, y la escasa literatura científica sobre los efectos de las variables ambientales en el estado de salud.

Se puede concluir que se encontró asociación entre la presencia del riesgo de depresión en los adultos mayores, principalmente en aquellos mayores de 75 años, y el sexo femenino, la residencia en la zona rural, la viudez, el riesgo de ansiedad, la capacidad funcional moderada para realizar las actividades de la vida diaria y el alto riesgo de desnutrición.

El 7,9 \% de la variabilidad del riesgo de depresión en los adultos mayores se explicó por variables de contexto relacionadas con el municipio de residencia: el 5,0 \% de esta variación se relacionó con las condiciones de la manzana dentro del municipio, y el 87,1\% se atribuyó a variables de persona.

La medición de la prevalencia de depresión mediante la utilización de encuestas de población es una estrategia útil, ya que la estimación basada en los datos de los servicios de salud suele subestimar la magnitud del problema debido a que solo reflejan la proporción de individuos que después de un proceso complejo (33) han accedido efectivamente a los servicios de salud; por lo tanto, el tener en cuenta que algunos de los problemas de salud pueden explicarse con base en otras condiciones no individuales, como el sitio de residencia, es una alternativa que debe explorarse para mejorar los programas y las intervenciones en las comunidades.

\section{Agradecimientos}

Las investigadoras agradecen a los adultos mayores encuestados, a las instituciones financiadoras, y a los auxiliares, estudiantes y encuestadores que hicieron posible la realización de este proyecto.

\section{Conflicto de intereses}

Los autores declaran que no existió conflicto de intereses en lo concerniente a las partes involucradas en el convenio, los adultos mayores encuestados ni las entidades financiadoras.

\section{Financiación}

Este proyecto de investigación se ejecutó mediante un convenio de asociación entre el departamento de Antioquia, la Secretaría Seccional de Salud y Protección Social de Antioquia, y la Facultad de Medicina de la Universidad CES (2012cf164156). 


\section{Referencias}

1. Organización Mundial de la Salud. Envejecimiento activo: un marco político. Rev Esp Geriatr Gerontol. 2002;37:74-105.

2. Cardona D, Agudelo HB. La flor de la vida: pensemos en el adulto: aspectos de la calidad de vida de la población adulta: comparativo por sexo, Medellín, 2005. Medellín: Universidad de Antioquia; 2006.

3. De la Serna I. Salud mental en el anciano. Identificación y cuidados de los principales trastornos. Madrid: Instituto de Salud Pública; 2003. Fecha de consulta: 10 de octubre de 2014. Disponible en: http://www.inger.gob.mx/ bibliotecageriatria/acervo/pdf/saludmental.pdf.

4. Erikson E. Identidad, juventud y crisis. Buenos Aires: Editorial Paidós; 1968.

5. Villar F. El enfoque del ciclo vital: hacia un abordaje evolutivo del envejecimiento. En: Pinazo S, Sánchez M, editores. Gerontología: actualización, innovación y propuestas. Madrid: Prentice-Hall (Pearson Educación); 2005. p. 147-81.

6. Pérez VT, Arcia N. Comportamiento de los factores biosociales en la depresión del adulto mayor. Rev Cubana Med Gen Integr. 2008;24:1-20.

7. Federación Mundial de Salud Mental. La salud mental y el envejecimiento. Psiquiat Pública. 1999;11:169-75.

8. Castro M, Ramírez S, Aguilar M, Díaz V. Factores de riesgo asociados a la depresión del adulto mayor. Rev Neurol Neurocic Psiquiatr. 2006;39:132-7.

9. Bacca AM, González A, Uribe AF. Validación de la Escala de Depresión de Yesavage (versión reducida) en adultos mayores colombianos. Pensamiento Psicológico. 2005;1:5363.

10. Cardona D, Segura AM, Garzón MO. Situación de salud y condiciones de vida del adulto mayor. Departamento de Antioquia 2012. Medellín: Universidad CES \& Gobernación de Antioquia; 2013.

11. Yesavage JA, Brink TL. Development and validation of a geriatric depression screening scale: A preliminary report. $\mathrm{J}$ Psychiat Res. 1983;17:37-49.

12. Organización Panamericana de la Salud. Evaluación del estado mental y emocional del adulto mayor. Módulo 4. En: Módulos de valoración clínica. Parte I. Washington DC: OPS/ OMS. Fecha de consulta: 16 de octubre de 2014. Disponible en: http://www.sld.cu/galerias/pdf/sitios/gericuba/modulo4.pdf.

13. Goldberg D, Bridges K, Duncan-Innes P, Grayson D. Detecting anxiety and depression in general medical settings. Br Med J. 1988;97:897-9.

14. Katz S, Ford AB, Moskowitz RW, Jackson BA, Jaffe MW. Studies of illness in the aged. The index of ADL: A standardized measure of biological and psychosocial function. JAMA. 1963;185:914-9. http://dx.doi.org/10.1001/ jama.1963.03060120024016

15. American Academy of Family Physicians, American Dietetic Association and National Council on the Aging. Nutrition screening initiative. DETERMINE your nutritional health checklist. Nutrition Interventions Manual for Professionals Caring for Older Americans. Washington DC: AAFP; 1991.

16. Centro Latinoamericano y Caribeño de Demografía. Manual sobre indicadores de calidad de vida en la vejez. Santiago de Chile: CELADE/CEPAL; 2006.
17. Rasbash J, Charlton C, Jones K, Pillinger R. Manual Supplement for MLwiN version 2.10. United Kingdom: Centre for Multilevel Modelling, University of Bristol; 2009

18. Organización Mundial de la Salud. Carga de los trastornos mentales y conductuales. En: Informe sobre la salud en el mundo 2001. Ginebra: OMS; 2001. p. 19-46.

19. Medina-Mora ME, Borges G, Lara-Muñoz C, Benjet C, Blanco-Jaimes J, Feliz-Bautista C, et al. Prevalencia de trastornos mentales y uso de servicios: resultados de la Encuesta Nacional de Epidemiología Psiquiátrica en México. Salud Ment. 2003;26:1-16.

20. Tapia-Mejía MS, Morales-Hernández J, Cruz-Ortega R, De la Rosa-Morales V. Depresión en el adulto mayor con enfermedad crónica. Rev Enferm IMSS. 2000;8:81-6.

21. Estrada A, Cardona D, Segura AM, Ordóñez J, Osorio JJ, Chavarriaga LM. Síntomas depresivos en adultos mayores institucionalizados y factores asociados. Universitas Psychologica. 2013;12:81-94.

22. Tuesca R, Fierro N, Molinares A, Oviedo F, Polo Y, Polo J, et al. Los grupos de socialización como factor protector contra la depresión en personas ancianas: Baranquilla, Colombia. Rev Esp Salud Pública. 2003;77:595-604.

23. Caraveo J, Martínez N, Rivera B, Polo A. Prevalencia en la vida de episodios depresivos y utilización de servicios especializados. Salud Ment. 1997;20:15-23.

24. Patten SB, Sedwack B, Russel ML. Major depression: Prevalence, treatment utilization and age in Canada. Can $\mathrm{J}$ Clin Pharmacol. 2001;8:133-8.

25. Gómez-Restrepo C, Bohórquez A, Pinto-Masis D, GilLaverde JFA, Rondón-Sepúlveda M, Díaz-Granados N. Prevalencia de depresión y factores asociados con ella en la población colombiana. Rev Panam Salud Pública. 2004;16: 378-86. http://dx.doi.org/10.1590/S1020-49892004001200003

26. Ávila-Funes J, Garant M, Aguilar-Navarro S. Relación entre los factores que determinan los síntomas depresivos y los hábitos alimentarios en adultos mayores de México. Rev Panam Salud Pública. 2006;19:321-30. http://dx.doi. org/10.1590/S1020-49892006000500005

27. Urbina JR, Flores JM, García MP, Torres L, Torrubias RM. Síntomas depresivos en personas mayores. Prevalencia y factores asociados. Gac Sanit. 2007;21:37-42.

28. Ávila-Funes JA, Melano-Carranza E, Payette H, Amieva H. Síntomas depresivos como factor de riesgo de dependencia en adultos mayores. Salud Pública Mex. 2007;49:367-75.

29. Zung WW, Broadhead WE, Roth ME. Prevalence of depressive symptoms in primary care. J Family Practice. 1993;37:337-44.

30. Vink D, Aartsen MJ, Schoevers RA. Risk factors for anxiety and depression in the elderly: A review. J Affect Disord. 2008;106:29-44. http://dx.doi.org/10.1016/j.jad.2007.06.005

31. Balfour JL, Kaplan GA. Neighborhood environment and loss of physical function in older adults: Evidence from the Alameda county study. Am J Epidemiol. 2002;155:507-15. http://dx.doi.org/10.1093/aje/155.6.507

32. Weich S, Holt G, Twigg L, Jones K, Lewis G. Geographic variation in the prevalence of common mental disorders in Britain: A multilevel investigation. Am J Epidemiol. 2003;157:730-7. http://dx.doi.org/10.1093/aje/kwg035

33. Belló M, Puentes-Rosas E, Medina-Mora ME, Lozano R. Prevalencia y diagnóstico de depresión en población adulta en México. Salud Pública Mex. 2005;47:4-11. 